\title{
Validation of a Wearable Biometric System's Ability to Monitor Heart Rate in Two Different Climate Conditions under Variable Physical Activities
}

\author{
Chady Al Sayed, Ludwig Vinches, Stéphane Hallé \\ Department of Mechanical Engineering, École de Technologie Supérieure, Montreal, Canada \\ Email: chady.al-sayed.1@ens.etsmtl.ca
}

How to cite this paper: Al Sayed, C., Vinches, L. and Hallé, S. (2017) Validation of a Wearable Biometric System's Ability to Monitor Heart Rate in Two Different Climate Conditions under Variable Physical Activities. E-Health Telecommunication Systems and Networks, 6, 19-30. https://doi.org/10.4236/etsn.2017.62002

Received: May 16, 2017

Accepted: June 12, 2017

Published: June 15, 2017

Copyright $\odot 2017$ by authors and Scientific Research Publishing Inc. This work is licensed under the Creative Commons Attribution International License (CC BY 4.0).

http://creativecommons.org/licenses/by/4.0/

\begin{abstract}
Research has proven the importance of cooling garments in reducing heat stress, especially for workers in extreme environments. The currently available cooling capacity of these garments should be controlled for improving their efficiency and autonomy. In this study, we investigated the Hexoskin wearable biometric shirt's capacity to monitor heart rate. Twelve male volunteers wore a Hexoskin biometric shirt and Polar ${ }^{\circledast} \mathrm{H} 7$ heart rate sensor and they completed two identical tests under two different climate conditions $\left(25^{\circ} \mathrm{C} \pm\right.$ $0.5^{\circ} \mathrm{C} ; 39 \% \pm 1 \%$ relative humidity and $31^{\circ} \mathrm{C} \pm 0.5^{\circ} \mathrm{C} ; 60 \% \pm 1 \%$ relative humidity). The results from four different statistical methods show a high correlation and an absence of significant differences between the Polar ${ }^{\otimes}$ and Hexoskin systems in monitoring the subjects' heart rates. The Hexoskin wearable biometric shirt can be used to monitor the heart rate of humans in moderate or hot and humid climates under variable physical activities, regardless of their age, weight or height.
\end{abstract}

\section{Keywords}

Hexoskin Biometric System, Heart Rate, Hot and Humid Environments, Physical Activity

\section{Introduction}

Workers, such as miners, who are exposed to hot and humid environments, can suffer from symptoms and diseases related to heat stress [1]. These conditions can increase the risk of work accidents and even lead to death. Personal cooling garments can be worn [2] for minimizing heat stress, especially in places where 
effective air conditioning is not provided. A valid number of researchers have concluded that by controlling the cooling load of these garments, this would improve their efficiency and increase their autonomy [3] [4]. The control can be based on the user's physiological parameters, such as skin temperature and heart rate. In 2015, Villar et al. [5], validated the ability of a wearable biometric shirt, the Hexoskin [6], to monitor physical parameters during daily activities, such as walking, laying down and standing. The same year, Montes et al. [7] stated that there may be issues concerning the Hexoskin wearable system's ability to measure and record data in real-life settings after having tested it on ten subjects in trail-hiking conditions. They recommended that the system should be validated before being utilized in real-life scenarios.

The Hexoskin wearable shirt has not yet been validated for intense physical activities, such as athletic training [8] and mining tasks. Therefore, this study validated its ability to monitor heart rate during variable physical activities before the Hexoskin wearable shirt to be used for controlling the cooling ability of personal cooling garments. Moreover, the study included twelve male subjects which were firstly tested in a moderate climate $\left(25^{\circ} \mathrm{C} \pm 0.5^{\circ} \mathrm{C} ; 39^{\circ} \mathrm{C} \pm 1^{\circ} \mathrm{C}\right.$ relative humidity) and afterwards in a hot and humid climate $\left(31^{\circ} \mathrm{C} \pm 0.5^{\circ} \mathrm{C} ; 60 \% \pm\right.$ $1 \%$ relative humidity).

\section{Materials and Methods}

This study was designed to validate the Hexoskin wearable biometric shirt's capacity to monitor heart rate during variable physical activities, ranging from sitting to high-intensity workouts, using a stationary training bicycle. The test procedure was conducted under two different climate conditions.

\subsection{Subjects}

Twelve male subjects were recruited for this study. Their physical characteristics appear in Table 1. All subjects were informed about the purpose of the study, the experimental protocol and the risks related to the tests and each person completed a consensus letter before participating. This study was reviewed and approved by the École de Technologie Supérieure Ethics Committee (ethical reference code: H20160101). The body mass index was calculated by dividing the weight (in kilograms) by the height squared (in meters) for each subject.

\subsection{Measurement Instruments}

All twelve subjects wore biometric shirts suited to their thorax and hip measurements. Beneath the shirt, each participant wore a Polar ${ }^{\otimes} \mathrm{H} 7$ heart sensor, which has been used in many monitoring studies for its accuracy to detect and record the heart rate in real time [9] [10]. The sensor was attached to an adjustable strap and connected by Bluetooth to a Polar ${ }^{\circledR}$ M400 watch [11] worn on the subject's left hand.

Each subject's heart rate data was recorded simultaneously by the Hexoskin wearable biometric shirt and the Polar ${ }^{\circledast}$ M400 watch and then sent to a personal 
computer after each test. A Northern Lights Commercial UG-7020 Upright Cycle (Northern Lights Fitness Products, Cornwall, ON, Canada) was placed inside a climate chamber and used during the tests. Figure 1 shows a subject wearing the Hexoskin biometric shirt, the Polar ${ }^{\circledR} \mathrm{H} 7$ heart rate sensor and the Polar ${ }^{\circledast}$ M400 watch.

\subsection{Climate Conditions}

All twelve subjects completed the experimental protocol in two different climate environments. The first had a moderate climate with a dry bulb temperature of $25^{\circ} \mathrm{C} \pm 0.5^{\circ} \mathrm{C}$; and a relative humidity of $39^{\circ} \mathrm{C} \pm 1^{\circ} \mathrm{C}$. The second had a hot and humid climate, with a dry bulb temperature of $31^{\circ} \mathrm{C} \pm 0.5^{\circ} \mathrm{C}$; and relative humidity of $60 \% \pm 1 \%$.

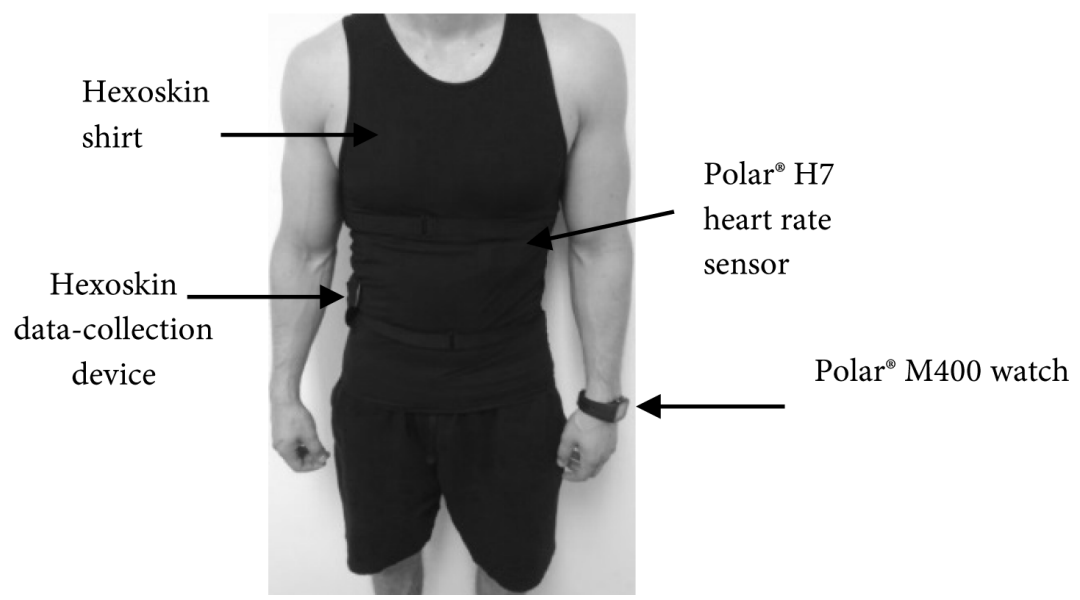

Figure 1. Subject wearing Hexoskin and Polar ${ }^{\circledast}$ devices.

Table 1. Physical characteristics of subjects.

\begin{tabular}{ccccc}
\hline Subject & Age (years) & Weight $(\mathrm{kg})$ & Height $(\mathrm{m})$ & Body mass index $\left(\mathrm{kg} / \mathrm{m}^{2}\right)$ \\
\hline 1 & 30 & 92.0 & 1.89 & 25.8 \\
2 & 22 & 75.0 & 1.78 & 23.7 \\
3 & 23 & 74.5 & 1.80 & 23.0 \\
4 & 22 & 75.0 & 1.76 & 24.2 \\
5 & 27 & 78.5 & 1.81 & 24.0 \\
6 & 25 & 66.5 & 1.70 & 22.9 \\
7 & 25 & 74.0 & 1.69 & 25.9 \\
8 & 25 & 59.0 & 1.77 & 18.8 \\
9 & 19 & 72.0 & 1.70 & 24.9 \\
10 & 23 & 77.0 & 1.80 & 23.8 \\
11 & 24 & 83.0 & 1.71 & 28.4 \\
12 & 33 & 86.0 & 1.78 & 27.1 \\
Arithmetic & $24.8 \pm 3.8$ & $76.0 \pm 8.6$ & $1.77 \pm 0.06$ & $24.4 \pm 2.4$ \\
\hline & & & &
\end{tabular}




\subsection{Test Procedure}

Prior to testing, every subject was again informed of the experimental protocol and climate conditions. Each subject completed two tests using the same experimental protocol, but under two different climate environments. The subjects were required to wait at least three days between the two tests. All the subjects were informed not to drink any caffeine or energy drinks four hours before the tests. Six subjects started their tests in a moderate climate while the other half, in a hot and humid environment.

Once the subjects arrived at the laboratory, before beginning the first test, their weight and height were measured and their body mass index was calculated. After that, the subjects first put on the Polar ${ }^{\circledR} \mathrm{H} 7$ heart rate sensor and the Polar ${ }^{\oplus}$ M400 watch and then the Hexoskin biometric shirt without connecting the Hexoskin data-collection device. The Hexoskin shirt comes with two elastic bands, which must be fastened around the torso to ensure that the sensors in the Hexoskin shirt are always in direct contact with the subject's skin (see Figure 1). All Hexoskin sensors were well moistened before each test. Both the Hexoskin and Polar ${ }^{\oplus} \mathrm{H} 7$ heart rate sensors are usually positioned in the same place, just below the chest muscles. To avoid any interference between the two sensors, the Polar ${ }^{\oplus}$ H7 heart rate sensor was placed lower on the torso than the Hexoskin sensor by two centimeters for all the tests conducted in this study. Once the equipment had been inspected on each subject, each person entered the climate chamber along with the researcher. Each subject mounted the upright cycle. Then, the researcher connected the Hexoskin data-collecting device to the Hexoskin shirt of the subject and started the Polar ${ }^{\oplus}$ M400 watch. During all the tests, each subject was given $500 \mathrm{ml}$ of water, which he could drink at any time during the test. If the heart rate of any subject during the physical activities reaches $90 \%$ of its average maximum heart rate recommended by the American Heart Association $^{\oplus}$ [12], the test will be stopped immediately and the subject will remain in his seat for 2 minutes, then will be accompanied outside the climate chamber for rest, till his heart rate decreases to its average resting rate (between 60 and 100 beats per minute) before leaving the laboratory.

The experimental protocol lasted a total of twenty-eight minutes as indicated in Figure 2. In the first minute (acclimatization stage), the subject sits on the

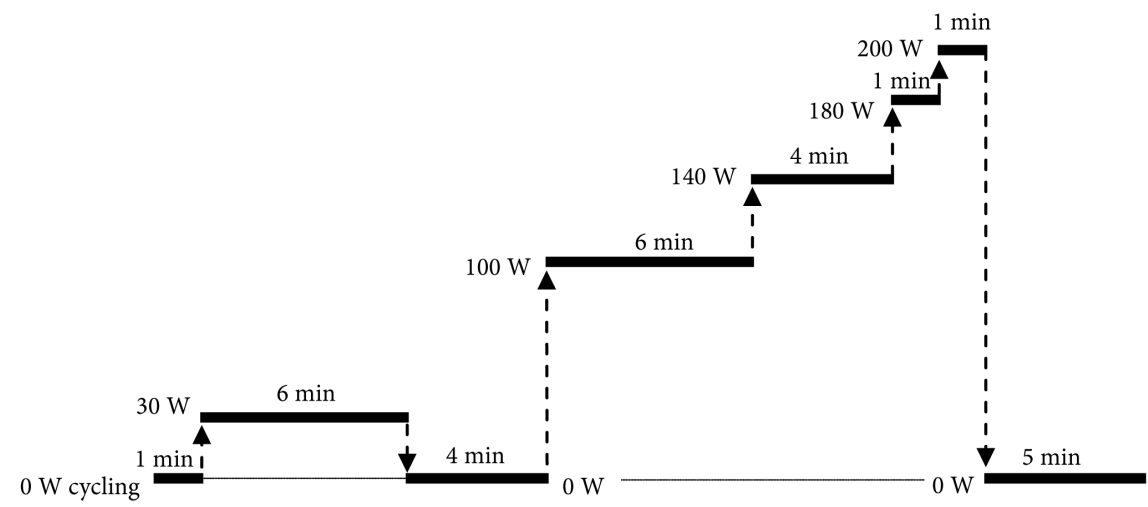

Figure 2. Experimental protocol. 
upright cycle without doing any exercise. After the first minute, the subject starts cycling to a level of $30 \mathrm{~W}$ and remains at that level for six minutes (warm-up stage). After the six minutes, the subject stops cycling and rests in the same position for four minutes. After the four-minute rest, the subject starts cycling at a level of $100 \mathrm{~W}$ and maintains this intensity for six minutes. The level is then increased and the subject maintains a $140 \mathrm{~W}$ level for four minutes. Again, the cycling level is increased and the subject maintains a cycling power of $180 \mathrm{~W}$ for one minute, followed by a $200 \mathrm{~W}$ cycling effort for the last minute. The subject then stops cycling and rests for five minutes in the same position, without leaving the climate chamber. After the five-minute rest, the Polar ${ }^{\circledR}$ M400 watch is turned off, and the Hexoskin data-collection device is disconnected. Figure 2 shows the experimental protocol used on all subjects under the two climates.

The subject leaves the climate chamber and is asked to rest for an additional ten minutes before leaving the laboratory, to ensure he is in healthy condition. After completing his first test, the subject was required to wait a minimum of three days before doing his second test. All data collected by the Polar ${ }^{\circledR}$ M400 watch and the Hexoskin data-collection device were transferred to a personal computer after each test.

\subsection{Data Analysis}

The collected data was analyzed using STATA $/$ SE (version 11.2), a data analysis and statistical software (StataCorp LP, Texas, USA). We started by comparing the degree of correlation between the two devices (Hexoskin and Polar ${ }^{\circledR}$ ) and for each level of effort. In this first approach, we tried to ascertain whether the changes that occur in the collected data of the first variable (Polar $\left.{ }^{\circledR}\right)$ affect in a predictable way, the changes that occur in the data collected by the second variable (Hexoskin). This evaluation would also provide an estimation of the highest and lowest percentage of correlation between the two devices, and at which level of exertion or climate condition. A degree of correlation higher than $0.70(70 \%)$ was considered to be positively correlated and the null hypothesis (equality of means of heart rates reported by the two devices) would not be rejected under this circumstances [13].

Then, the data were analyzed using the Wilks' lambda test of equality of means [14]. This statistical method checked for any significant differences between the mean values of the collected data (of the 12 subjects) from the two devices, at any level of exertion or under any climate, but without taking into consideration the physical characteristics of the subjects, such as their weight and height. A p-value equal to or less than 0.05 would indicate a significant difference in the mean values.

A third analysis was conducted by using the ANOVA method with repeated data. The physical characteristics of the subjects were considered in order to see if they affected the correlations between the two devices and if any significant differences appeared ( $\mathrm{p}$-value $\leq 0.05$ ).

Finally, the linear regressions method with mixed data was used for examin- 
ing the switching of the devices (from Polar ${ }^{\circledast}$ to Hexoskin and vice-versa) from one level of exertion to the next. The previous switching was checked relevant to exhibiting any significant differences between the data collected by the two devices, by taking into consideration the physical characteristics of the subjects.

\section{Results}

The validity of the Hexoskin wearable biometric system to monitor heart rate was assessed in four different ways using various levels of physical exertion, which were determined by the effort made by the subject during the tests. Table 2 shows the physical effort and each level's duration.

The first step in analyzing the validity of the Hexoskin wearable biometric system was to test whether the variations of the mean values collected by the Hexoskin are predictable using the variations that were captured by the Polar ${ }^{\circ}$ device. The results are displayed in Table 3 and are associated to the method comparing the degree of correlation. The degree of correlation between the two devices has a minimum of $0.9162(91.62 \%)$ for level 1 in a moderate climate and a maximum of 0.9995 (99.95\%) for level 5 in a hot and humid climate. The average degree of correlation in the moderate climate is 0.9987 (99.87\%) and in the hot and humid climate, it is 0.9985 (99.85\%).

Table 2. Physical effort and its duration for each level.

\begin{tabular}{cccc}
\hline Levels & Physical effort (watts) & Duration (minutes) & Status \\
\hline 1 & 0 & 1 & Acclimatization \\
2 & 30 & 6 & Warm-up \\
3 & 0 & 4 & Rest \\
4 & 100 & 6 & Low effort \\
5 & 140 & 4 & Medium effort \\
6 & 180 & 1 & Intense effort \\
7 & 200 & 1 & Very intense effort \\
8 & 0 & 5 & Recovery \\
\hline
\end{tabular}

Table 3. Results of comparison of the degree of correlation between the two devices.

\begin{tabular}{ccc}
\hline Levels & $\begin{array}{c}\text { Correlation between Hexoskin and } \\
\text { Polar }^{\circledR} \text { (Moderate climate) }\end{array}$ & $\begin{array}{c}\text { Correlation between Hexoskin and Polar } \\
\text { (Hot and humid climate) }\end{array}$ \\
\hline 1 & 0.9162 & 0.9611 \\
2 & 0.9844 & 0.9817 \\
3 & 0.9848 & 0.9863 \\
4 & 0.9981 & 0.9977 \\
5 & 0.9993 & 0.9995 \\
6 & 0.9992 & 0.9993 \\
7 & 0.9991 & 0.9992 \\
8 & 0.9991 & 0.9991 \\
Average & 0.9987 & 0.9985 \\
\hline
\end{tabular}


For a clearer visual comparison, the charts below present the mean values of the heart rates detected by the two devices, averaged every minute, for all the subjects in the moderate climate (Figure 3(a)) and in the hot and humid climate (Figure 3(b)).

A clear indication of the high correlation between the two data collected from the two devices is the overlapping of the two curves in each line diagram. For all the line diagrams and climates, the low physical effort related to level $1(0 \mathrm{~W}), 2$ $(30 \mathrm{~W})$ and $3(100 \mathrm{~W})$ has little effect on the heart rate, as seen by the diagram's tendency to be a straight line between minute 1 and minute 11 (level 1, 2 and 3). However, between minutes 11 and 17 (level 4), even though the physical effort

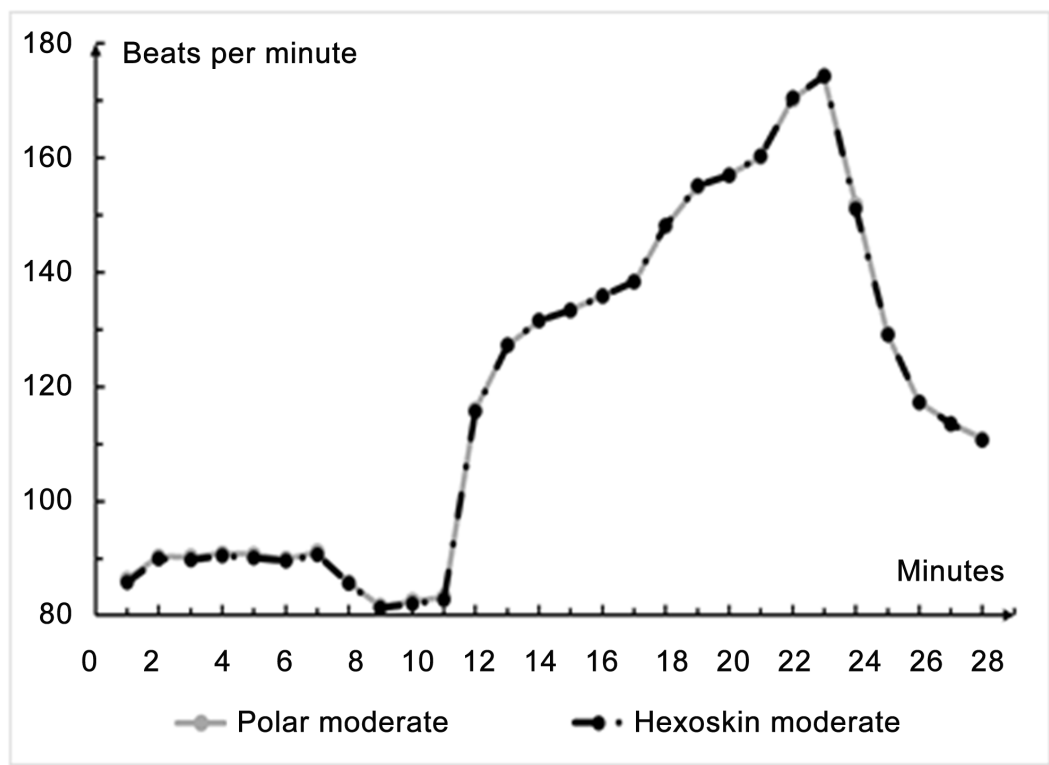

(a)

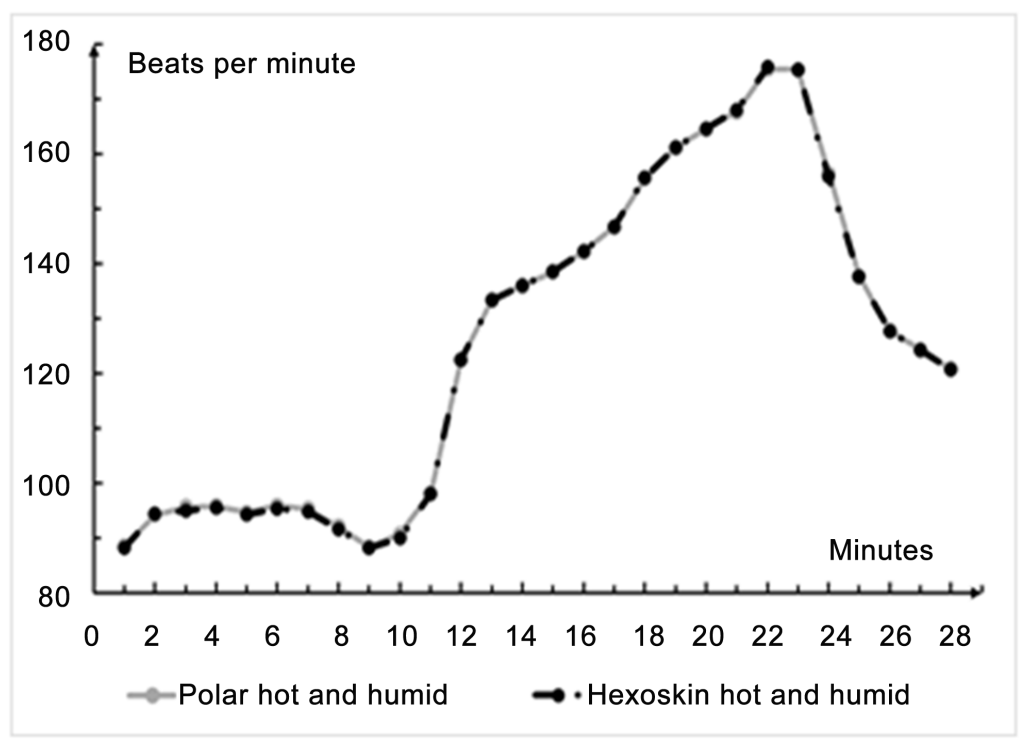

(b)

Figure 3. (a) Average heart rates detected by the two devices in moderate climate; (b) average heart rates detected by the two devices in hot and humid climate. 
was maintained at $100 \mathrm{~W}$, the curves continue to increase. Finally, after minute 21 , even a small increase in physical effort (increase of $40 \mathrm{~W}$ from minute 21 to 22 , and $20 \mathrm{~W}$ from minute 22 to 23 ) led to significant heart rate raises, which are visible in the two diagrams as the important positive slopes occurring between minutes 21 and 23 (level 6 and 7). Although the two diagrams (in moderate, in hot and humid climates) are not exactly similar in shape, they reach the same peek after minute 23 , which corresponds to approximately 175 beats per minute.

The results of the analysis using the Wilks' lambda test of equality of means are listed in Table 4. The p-values associated with the $\mathrm{F}$ test (probability $>\mathrm{F}$ ) were always superior to 0.05 . The two lowest $\mathrm{p}$-values occurred for the same level of exertion (level 2). In the moderate climate, p-value was 0.3467 and in the hot and humid climate, it had the value of 0.6336 . The average p-value was 0.9967 in the moderate climate and 0.9988 in the hot and humid climate.

When we took into consideration the physical characteristics of the subjects, such as their weight, height, age and body mass index, while analyzing the data, by using the ANOVA method with repeated data, we found the results listed below in Table 5. The p-value while comparing the data collected by the two devices and under the same level of exertion had an average of 0.9934 . We also

Table 4. Results from the Wilks' lambda test of equality of means.

\begin{tabular}{ccccc}
\hline Climate & \multicolumn{2}{c}{ Moderate } & \multicolumn{2}{c}{ Hot and humid } \\
\hline Levels & F value & Probability $>$ F & F value & Probability $>$ F \\
\hline 1 & 0.24 & 0.9451 & 0.05 & 0.9981 \\
2 & 1.12 & 0.3467 & 0.69 & 0.6336 \\
3 & 0.27 & 0.9314 & 0.66 & 0.6527 \\
4 & 0.02 & 0.9999 & 0.00 & 1.0000 \\
5 & 0.02 & 0.9999 & 0.02 & 0.9998 \\
6 & 0.02 & 0.9999 & 0.03 & 0.9994 \\
7 & 0.07 & 0.9964 & 0.09 & 0.9935 \\
8 & 0.02 & 0.9998 & 0.03 & 0.9995 \\
Average & 0.07 & 0.9967 & 0.04 & 0.9988 \\
\hline
\end{tabular}

Table 5. Results from the ANOVA analysis with repeated data.

\begin{tabular}{cccc}
\hline Heart rate & Mean squared & F value & Probability $>$ F \\
\hline Polar ${ }^{\oplus}$ versus Hexoskin (same level) & 0.22 & 0.00 & 0.9934 \\
Moderate versus hot and humid & 14235.02 & 4.38 & 0.0428 \\
Polar $^{\circledast}$ versus Hexoskin (same climate) & 2.00 & 0.00 & 0.9803 \\
Adding ages & 1960.31 & 0.60 & 0.4421 \\
Adding heights & 1667.76 & 0.51 & 0.4781 \\
Adding weights & 1613.66 & 0.50 & 0.4853 \\
Adding body mass indexes & 1425.61 & 0.44 & 0.5118 \\
\hline
\end{tabular}


found that if we compare the data collected in moderate and in hot and humid climates, the average p-value decreases until 0.0428 . The $\mathrm{p}$-value while comparing the data collected by the two devices and under the same climate had an average of 0.9803 . After adding the physical characteristics of the subjects, the pvalues for the same comparison were 0.4421 (after adding the ages), 0.4781 (after adding the heights), 0.4853 (after adding the weights) and 0.5118 (after adding the body mass indexes).

Finally, the linear regression with mixed data method was used. The results are listed in Table 6. We found that the lowest $\mathrm{p}$-value associated to the $\mathrm{z}$ value (Probability $>\mathrm{z}$ ) was 0.693 . This value was obtained by going from the first level of exertion to the second and by switching devices (from Polar ${ }^{\oplus}$ to Hexoskin). Any p-value equal to or less than 0.05 would reveal a significant difference. The highest p-value found was 0.824 associated to the transition from level 2 to level 3. Even after taking into consideration the physical characteristics of the subjects, the p-value was never less than 0.602 (by adding ages) and rose to 0.971 after adding the body mass indexes.

\section{Discussion}

The capacity of the wearable biometric system (Hexoskin) to monitor heart rate in two different climate conditions and under variable physical activities was assessed using four different statistical methods. The results of correlations between the Polar ${ }^{\circledR}$ and Hexoskin systems, using the Karl Pearson's coefficient, show that the two devices are highly similar in terms of performance in measuring heart rates, under the two climate conditions and the eight levels of physical exertion (correlation degrees $>0.70$ ). The average correlation percentage was almost 99.8\%. This first conclusion is also visible in Figure 3(a) and Figure 3(b) where each diagram (representing the data collected by the Hexoskin and Polar systems during all the tests) clearly shows the overlapping of the two curves.

Table 6. Results from the linear regressions with mixed data method.

\begin{tabular}{ccccc}
\hline Heart rate & Coefficient & Standard error & z value & Probability $>$ z \\
Levels 1 - 2 & 2.08 & 5.28 & 0.39 & 0.693 \\
Levels 2 - 3 & 1.19 & 5.38 & 0.22 & 0.824 \\
Levels 3 - 4 & 1.38 & 5.20 & 0.27 & 0.790 \\
Levels 4 - 5 & 1.82 & 5.38 & 0.34 & 0.735 \\
Levels 5 - 6 & 1.91 & 6.81 & 0.28 & 0.779 \\
Levels 6 - 7 & 2.25 & 6.81 & 0.33 & 0.741 \\
Levels 7 - 8 & 1.62 & 5.20 & 0.31 & 0.755 \\
Adding ages & 0.29 & 0.56 & 0.52 & 0.602 \\
Adding heights & -78.84 & 383.31 & -0.21 & 0.837 \\
Adding weights & 0.15 & 4.39 & 0.04 & 0.971 \\
Adding body mass indexes & -3.61 & 13.48 & -0.27 & 0.789 \\
\hline
\end{tabular}


Using the Wilks' lambda test of equality of means, we found that Hexoskin and Polar ${ }^{\oplus}$ had the same means when the same climate condition was considered. This result remaind valid for each of the eight levels of the experiment. Indeed, in Table 4, the p-values associated with the F test were always superior to 0.05 which led us not to reject the null hypothesis of equality of means. The results from the ANOVA analysis displayed in Table 5 showed that the two devices did not have significant differences in influencing the variations of heart rates when we checked for the physiological characteristics of each subject and the climatic conditions of the experiment. Indeed, the high p-value associated to the $\mathrm{F}$ test of the variance between the two devices (0.9934) did not allow for the rejection of the null hypothesis of difference's absence between Polar ${ }^{\circledR}$ and Hexoskin. Even when considering the ages, weights, heights and body mass indexes of all the subjects, the associated p-values were always greater than 0.05 and were respectively, $0.4421,0.4853,0.4781$ and 0.5118 . Also, when the mean values were compared under different climates, it was found that there was not significant difference ( $\mathrm{p}$-value $=0.0428$ ), which was expected as the subjects did not have the same heart rates for a same given level, under two different climates [15]. That is why we can clearly understand that the mean values in Figure 3(a) are always lower than those in Figure 3(b) at the same level of exertion (except for minute 23 , where they are equal).

Based on the results obtained by the linear regressions method with mixed data, the lowest $\mathrm{p}$-value (0.693) associated to the $\mathrm{Z}$ tests was also higher than 0.05 , which suggested that there was no significant difference when switching from Polar ${ }^{\circledast}$ to Hexoskin, and from one level of exertion to the next, under the same climate. After adding the physical characteristics of all subjects, we found that the calculated p-values were between 0.602 and 0.971 (larger than 0.05), which led us to the same conclusion as before. No significant differences appeared between Polar ${ }^{\oplus}$ and Hexoskin, when the levels of exertion changed under the same climate, regardless of the weight, age, height or body mass index of the subject.

\section{Conclusions}

The validity of the Hexoskin wearable biometric shirt to monitor heart rate in two different climate conditions and under variable physical activities was analyzed and assessed using four different statistical methods. They all concluded to the absence of significant differences between Polar ${ }^{\circledR}$ and Hexoskin in their respective capacities to record with accuracy of the heart rates for the same climate condition when taking into consideration certain physiological characteristics of the subjects.

Finally, we found that the Hexoskin wearable shirt can be used in both moderate and hot and humid climates to monitor the heart rate of any subject, regardless of his age, weight or height. It can also be used by athletes to monitor their heart rates under variable physical activities that range from rest to extreme physical exertion. 


\section{Acknowledgments}

The authors would like to thank the Fonds de Recherche du Québec-Nature et Technologies (FRQNT) for its financial contribution to this study.

\section{Conflicts of Interest}

The Hexoskin wearable biometric shirts used in this study were provided by Carré Technologies Inc. Carré Technologies Inc. had no role in developing, conducting or analyzing this study. The authors declare that they have no conflict of interest with the Hexoskin wearable biometric shirt and Polar ${ }^{\circledast}$ biometric systems used in this study.

\section{References}

[1] Michael Donoghue, A. (2004) Heat Illness in the U.S. Mining Industry. American Journal of Industrial Medicine, 45, 351-356. https://doi.org/10.1002/ajim.10345

[2] Al Sayed, C., Vinches, L. and Hallé, S. (2016) Towards Optimizing a Personal Cooling Garment for Hot and Humid Deep Mining Conditions. Open Journal of Optimization, 5, 35-43. https://doi.org/10.4236/ojop.2016.51005

[3] Cadarette, B.S., et al. (2006) Intermittent Microclimate Cooling during ExerciseHeat Stress in US Army Chemical Protective Clothing. Ergonomics, 49, 209-219. https://doi.org/10.1080/00140130500436106

[4] Vernieuw, C.R., Stephenson, L.A. and Kolka, M.A. (2007) Thermal Comfort and Sensation in Men Wearing a Cooling System Controlled by Skin Temperature. Human Factors. The Journal of the Human Factors and Ergonomics Society, 49, 1033-1044. https://doi.org/10.1518/001872007X249893

[5] Villar, R., Beltrame, T. and Hughson, R.L. (2015) Validation of the Hexoskin Wearable Vest during Lying, Sitting, Standing, and Walking Activities. Applied Physiology, Nutrition, and Metabolism, 40, 1019-1024.

https://doi.org/10.1139/apnm-2015-0140

[6] Technologies, C. Hexoskin Wearable Body Metrics. http://www.hexoskin.com/

[7] Montes, J., et al. (2015) Using Hexoskin Wearable Technology to Obtain Body Metrics in a Trail Hiking Setting. International Journal of Exercise Science, 8, 11.

[8] Düking, P., et al. (2016) Comparison of Non-Invasive Individual Monitoring of the Training and Health of Athletes with Commercially Available Wearable Technologies. Frontiers in Physiology, 7, 71.

[9] Li, C.-T., Cao, J. and Li, T.M.H. (2016) Eustress or Distress: An Empirical Study of Perceived Stress in Everyday College Life. Proceedings of the 2016 ACM International Joint Conference on Pervasive and Ubiquitous Computing Adjunct, Heidelberg, Germany, 12-16 September 2016, 1209-1217. https://doi.org/10.1145/2968219.2968309

[10] Cheatham, S.W., Kolber, M.J. and Ernst, M.P. (2015) Concurrent Validity of Resting Pulse-Rate Measurements: A Comparison of 2 Smartphone Applications, the Polar H7 Belt Monitor, and a Pulse Oximeter with Bluetooth. Journal of Sport Rehabilitation, 24, 171-178. https://doi.org/10.1123/jsr.2013-0145

[11] Polar. Polar M400-H7. https://www.polar.com/ca-en/products/sport/M400\#support_accessories

[12] American, H.A. http://www.heart.org/HEARTORG/HealthyLiving/PhysicalActivity/FitnessBasics/T 
arget-Heart-Rates_UCM_434341_Article.jsp\#.WRwgvsZlO70

[13] Montes, J. (2015) Validation and Reliability of the Hexoskin and FitBit Flex Wearable BIO Collection Devices, in Kinesiology and Nutrition Sciences. University of Nevada, Las Vegas, 19.

[14] Polit, D.F. (1996) Data Analysis \& statistics for Nursing Research. Appleton \& Lange.

[15] Galloway, S. and Maughan, R.J. (1997) Effects of Ambient Temperature on the Capacity to Perform Prolonged Cycle Exercise in Man. Medicine and Science in Sports and Exercise, 29, 1240-1249. https://doi.org/10.1097/00005768-199709000-00018

Submit or recommend next manuscript to SCIRP and we will provide best service for you:

Accepting pre-submission inquiries through Email, Facebook, LinkedIn, Twitter, etc. A wide selection of journals (inclusive of 9 subjects, more than 200 journals)

Providing 24-hour high-quality service

User-friendly online submission system

Fair and swift peer-review system

Efficient typesetting and proofreading procedure

Display of the result of downloads and visits, as well as the number of cited articles

Maximum dissemination of your research work

Submit your manuscript at: http://papersubmission.scirp.org/

Or contact etsn@scirp.org 